\title{
Correlation Inequalities and Equilibrium States. II ${ }^{\star}$
}

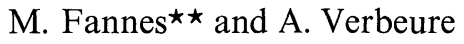 \\ Instituut voor Theoretische Fysica, Universiteit Leuven, B-3030 Leuven, Belgium
}

\begin{abstract}
The hierarchy of some relevant correlation inequalities is settled, together with their relation to equilibrium conditions.
\end{abstract}

\section{Introduction}

Recently it was proved that the Roepstorff upper bound [1] for what we call the Duhamel two-point function, defined for a time invariant state, is equivalent with the KMS-condition for equilibrium states [2]. Along the same lines Sewell [3] used an other inequality for correlation functions and proved that it yields the KMScondition. This technique showed to be a powerful method to prove the equivalence of KMS with local variation principles.

Here we give a proof of the equivalence of the two inequalities for time invariant states (Theorem II.4). Therefore for practical services the one or the other may be used. The Roepstorff upper bound seems to be more directly connected to physical quantities [4] whereas the other inequality looks interesting as it is linear in the Hamiltonian.

Furthermore recently a bunch of correlation inequalities has been derived for KMS-states [1-6]. Theorem II.4 yields now more elegant and more direct proofs of these inequalities. In this note we consider more five inequalities. It turns out that in general they are not equivalent to KMS or to the other conditions in Theorem II.4. Therefore the question of hierarchy between these inequalities is raised and solved.

\section{Equilibrium Conditions}

Let $\mathscr{M}$ be a Neumann algebra on a Hilbert space $\mathscr{H}$. Let $H$ be a self-adjoint operator on $\mathscr{H}$ and $U_{t}=\exp$ it $H, t \in \mathbb{R}$ such that $t \rightarrow U_{t} \cdot U_{t}^{*}$ is a group of automorphisms of $\mathscr{M}$. We consider the vector state $\omega(x)=(\Omega, x \Omega) x \in \mathscr{M}$ with $\Omega$ a cyclic element of $\mathscr{H}$, such that $\Omega \in \mathscr{D}(H)$.

$\star$ Dedicated to Professor L. P. Bouckaert on the occasion of his seventieth birthday

$\star \star \quad$ Aangesteld Navorser NFWO, Belgium 
Furthermore suppose that $\mathscr{M} \Omega$ belongs to the domain $\mathscr{D}\left(\Delta^{t / 2}\right)$ of $\Delta^{t / 2}=\exp (-t H / 2), t \in[0,1]$.

Definition II.1. The state $\omega$ on $\mathscr{M}$ is called KMS if $\omega$ satisfies : for all pairs $x, y$ in $\mathscr{M}$ :

$$
\left(\Delta^{1 / 2} x \Omega, \Delta^{1 / 2} y \Omega\right)=\left(x^{*} \Omega, y^{*} \Omega\right) .
$$

Definition II.2. The state $\omega$ on $\mathscr{M}$ satisfies the Roepstorff inequality if for all $x \in \mathscr{M}, \omega$ satisfies:

$(T x \Omega, T x \Omega) \leqq F\left(\omega\left(x^{*} x\right), \omega\left(x x^{*}\right)\right)$,

where

$$
\begin{aligned}
T & =\left(\frac{\Delta-1}{\ln \Delta}\right)^{1 / 2} \quad \text { (see [6] and [7]), } \\
F(u, v) & = \begin{cases}(u-v) /(\ln u-\ln v) & \text { if } u \neq v \\
u & \text { if } u=v .\end{cases}
\end{aligned}
$$

Definition II.3. The state $\omega$ on $\mathscr{M}$ satisfies the autocorrelation lower bound if for all $x$ in $\mathscr{D}(H)$, domain of $H$ :

$$
x \Omega,[H, x] \Omega) \geqq \mathscr{G}\left(\omega\left(x^{*} x\right), \omega\left(x x^{*}\right)\right),
$$

where

$$
\mathscr{G}(u, v)= \begin{cases}u \ln u-u \ln v & \text { if } v>0, u>0 \\ 0 & \text { if } u=v=0\end{cases}
$$

Theorem II.4. The following conditions are equivalent :

(i) $\omega$ is KMS.

(ii) $\omega$ is stationary (i.e. $U_{t} \Omega=\Omega$ te $\mathbb{R}$ ) and satisfies (2).

(iii) $\omega$ is stationary and satisfies (3).

Proof. (i) implies (ii) see [6]. Now we prove (ii) $\rightarrow$ (iii):

Define implicitly the function $g: x \in \mathbb{R}^{+} \rightarrow g(x) \in \mathbb{R}$ by

$$
g\left(\frac{1-e^{-x}}{x}\right)=x \text {. }
$$

Let $T(x)=\frac{1-e^{-x}}{x}$ then $T^{-1}=g$.

Furthermore

$$
T(x)=\int_{0}^{1} d t e^{-x t}
$$

hence $T$ is a decreasing convex function on $\mathbb{R}$ with range $\mathbb{R}^{+}$, therefore $g$ shares this property.

Let $x \Omega$ be an element of the domain $\mathscr{D}(H)$ and $\{E(\lambda), \lambda \in \mathbb{R}\}$ the spectral resolution of $H$ then $\mu(\lambda)=\frac{\|E(\lambda) x \Omega\|^{2}}{\|x \Omega\|^{2}}$ is a probability measure; denote $X=\frac{\omega\left(x^{*} x\right)}{\omega\left(x x^{*}\right)}$ 
then the inequality in (ii) reads as:

$$
\int d \mu(\lambda) \frac{1-e^{-\lambda}}{\lambda} \leqq \frac{X-1}{X \ln X} .
$$

Applying the function $g$ yields:

$$
g\left(\int d \mu(\lambda) \frac{1-e^{-\lambda}}{\lambda}\right) \geqq \ln X .
$$

Using the convexity of $g$ and Jenssen's inequality we get

$$
\frac{(x \Omega, H x \Omega)}{\omega\left(x^{*} x\right)}=\int d \mu(\lambda) g(T(\lambda))=\int d \mu(\lambda) \lambda \geqq \ln X .
$$

Using the invariance of the state one gets (iii).

Finally (iii) $\rightarrow$ (i) is proved in [3].

\section{Hierarchy of Correlation Inequalities}

As it is possible to settle this question for matrix algebras we limit ourself to this situation. The statements below can be generalized to the general situation. Therefore let $M_{n}$ be the set of all $n \times n$ complex matrices $n \in 2,3,4, \ldots, H=H^{*} \in M_{n}$ and let $\omega$ be any state on $M_{n}$, i.e. $\omega(x)=\operatorname{Tr} \varrho x, x \in M_{n}$, where $\varrho$ is any density matrix on $C_{n}$ such that $[\varrho, H]=0$.

Theorem III.1. (i) Inequality (3) implies: for all $x \in M_{n}$ :

$$
\omega\left(\left[x^{*}, x\right]\right) \ln \frac{\omega\left(x^{*} x\right)}{\omega\left(x x^{*}\right)} \leqq \omega\left(\left[x^{*},[H, x]\right]\right) .
$$

(ii) Inequality (4) is equivalent to: for all $x, y \in M_{n}$ :

$$
\left|\omega\left(\left[x^{*}, y\right]\right)\right|^{2} \leqq \frac{\omega\left(\left[x, x^{*}\right]\right)}{\ln \omega\left(x x^{*}\right)-\ln \omega\left(x^{*} x\right)} \omega\left(\left[y^{*},[H, y]\right]\right) .
$$

(iii) Inequality (4) does not imply inequality (3).

Proof. (i) Inequality (4) is obtained from summing the inequalities (3) for $x$ and for $x^{*}$.

(ii) (4) is obtained from (5) by putting $y=x$.

Now we prove the converse.

From (4): for all $x, y \in M_{n}$ :

$$
(x, y)_{\sim}=\omega\left(\left[x^{*},[H, y]\right]\right)
$$

defines a positive sesquilinear form $(\cdot, \cdot)_{\sim}$ on $M_{n}$.

Let $L$ be the Liouville operator: $L x=[H, x], x \in M_{n}$.

One checks that for all $x, y \in M_{n}$ :

$$
\begin{aligned}
& (L x, y)_{\sim}=(x, L y)_{\sim} \\
& \left(x^{*}, y^{*}\right)_{\sim}=(y, x)_{\sim} .
\end{aligned}
$$


We use the following observation. Any element $x \in M_{n}$ can be written as:

$$
x=a+L b
$$

$a, b \in M_{n}, L a=0$. In this finite dimensional case such a decomposition is possible e.g. with respect to the trace (for the general case a more detailed analysis is needed). Let

$$
\mathscr{N}=\left\{x \in M_{n} \mid(x, x)_{\sim}=0\right\}
$$

then $\mathscr{N}$ is left invariant by $L$, as :

$$
\left|(L x, L x)_{\sim}\right|^{2} \leqq(x, x)_{\sim}\left(L^{2} x, L^{2} x\right)_{\sim} .
$$

Let $\mathscr{H}$ be the Hilbert space $M_{n} / \mathscr{N}$ with scalar product $(\cdot, \cdot)_{\sim}$ and $[\cdot]$ the canonical map of $M_{n}$ in $\mathscr{H}$.

a) Suppose $y \in \mathscr{N}$.

Remark first that if $L y=0$ then $\omega\left(\left[x^{*}, y\right]\right)=0$ for all $x \in M_{n}$. Indeed from (4): $\omega\left(y^{*} y\right)=\omega\left(y y^{*}\right)$.

By polarization

$$
\omega\left(\left[x^{*}, y\right]\right)=0 \text { for } \quad x, y \in \mathscr{N} .
$$

If $L a=0$, then $a^{*} \in \mathscr{N}$ and hence $\omega([y, a])=0$ for $y \in \mathscr{N}$.

In general let $x \in M_{n}$ then

$$
x^{*}=a+L b \quad \text { with } \quad L a=0
$$

and

$$
\omega\left(\left[y, x^{*}\right]\right)=\omega([y, a])+\omega([y, L b])
$$

the first term vanishes by the argument above, the second term vanishes because:

$$
|\omega([y, L b])|^{2}=\left|(y, b)_{\sim}\right|^{2} \leqq(y, y)_{\sim}(b, b)_{\sim}=0 .
$$

b) We have to treat the cases $\omega\left(x^{*} x\right)>\omega\left(x x^{*}\right)$ and $\omega\left(x^{*} x\right)<\omega\left(x x^{*}\right)$. We give in detail the first case. The second one is obtained by substituting $x$ into $x^{*}$ step by step. The case $\omega\left(x^{*} x\right)=\omega\left(x x^{*}\right)$ is obtained by continuity.

As $L$ is self-adjoint invertible on $\mathscr{H}$, for any element $x \in M_{n}$ we can write

$$
[x]=\sum_{k=1}^{r}\left[x_{k}\right]
$$

and

$$
x=\sum_{k=1}^{r} x_{k}+x_{0}
$$

with $L x_{k}=\lambda_{k} x_{k}, \lambda_{k} \neq 0, \lambda_{k} \neq \lambda_{l}$ for $k \neq l$

$$
\left(x_{k}, x_{k}\right)_{\sim} \neq 0, \quad\left(x_{0}, x_{0}\right)_{\sim}=0 \text {. }
$$


It follows that

$$
\begin{aligned}
& (x, x)_{\sim}=\sum_{k}\left(x_{k}, x_{k}\right)_{\sim} \\
& \omega\left(x_{k}^{*} x_{0}\right)=0 \\
& \omega\left(x_{k}^{*} x_{l}\right)=\delta_{k, l} \omega\left(x_{k}^{*} x_{k}\right)
\end{aligned}
$$

and therefore

$$
\begin{aligned}
& \omega\left(x^{*} x\right)=\sum_{k} \omega\left(x_{k}^{*} x_{k}\right)+\omega\left(x_{0}^{*} x_{0}\right) \\
& \omega\left(x x^{*}\right)=\sum_{k} \omega\left(x_{k} x_{k}^{*}\right)+\omega\left(x_{0} x_{0}^{*}\right) .
\end{aligned}
$$

Remark that by a) $\omega\left(x_{0}^{*} x_{0}\right)=\omega\left(x_{0} x_{0}^{*}\right)$.

For any element $y \in M_{n}$ : by a) and Schwartz inequality:

$$
\left.\left|\omega\left(\left[y^{*}, x\right]\right)\right|^{2}=\mid([y]), L^{-1}[x]\right)\left._{\sim}\right|^{2} \leqq([y],[y])_{\sim}\left(L^{-1}[x], L^{-1}[x]\right)_{\sim} .
$$

Remark that

$$
([y],[y])_{\sim}=\omega\left(\left[y^{*},[H, y]\right]\right)
$$

and

$$
\left(L^{-1}[x], L^{-1}[x]\right)_{\sim}=\sum_{k} \frac{1}{\lambda_{k}^{2}}\left(x_{k}, x_{k}\right)_{\sim} .
$$

From (4) applied to $x_{k}$ :

$$
\omega\left(\left[x_{k}^{*}, x_{k}\right]\right) \ln \frac{\omega\left(x_{k}^{*} x_{k}\right)}{\omega\left(x_{k} x_{k}^{*}\right)} \leqq \lambda_{k} \omega\left(\left[x_{k}^{*}, x_{k}\right]\right) .
$$

From the positivity of the right hand side, it follows that

$$
\lambda_{k} \ln \frac{\omega\left(x_{k}^{*} x_{k}\right)}{\omega\left(x_{k} x_{k}^{*}\right)} \geqq 0
$$

Dividing the inequality by this factor:

$$
\frac{\left(x_{k}, x_{k}\right)_{\sim}}{\lambda_{k}^{2}}=\frac{\omega\left(\left[x_{k}^{*}, x_{k}\right]\right)}{\lambda_{k}} \leqq \frac{\omega\left(\left[x_{k}^{*}, x_{k}\right]\right)}{\ln \omega\left(x_{k}^{*} x_{k}\right)-\ln \omega\left(x_{k} x_{k}^{*}\right)} .
$$

After the summation over $k$, using the joint convexity and the homogeneity of the function

$$
(u, v) \in\left(\mathbb{R}^{+}\right)^{2} \rightarrow \frac{u-v}{\ln u-\ln v}
$$


we get

$$
\begin{aligned}
\left(L^{-1}[x], L^{-1}[x]\right)_{\sim} & \leqq \frac{\omega\left(x^{*} x\right)-\omega\left(x x^{*}\right)}{\ln \frac{\omega\left(x^{*} x\right)-\omega\left(x_{0} x_{0}^{*}\right)}{\omega\left(x x^{*}\right)-\omega\left(x_{0} x_{0}^{*}\right)}} \\
& \leqq \frac{\omega\left(x^{*} x\right)-\omega\left(x x^{*}\right)}{\ln \omega\left(x^{*} x\right)-\ln \omega\left(x x^{*}\right)}
\end{aligned}
$$

(iii) Let $\omega$ be the trace state, then $\omega$ is a solution of (4), but in general not of (3). It is a solution of (3) if and only if $H$ is a constant.

Theorem III.2. (i) Inequality (5) implies:

for all $x, y \in M_{n}$ :

$$
|\omega([x, y])|^{2} \leqq \frac{1}{2} \omega\left(x x^{*}+x^{*} x\right) \omega\left(\left[y^{*},[H, y]\right]\right)
$$

(Bogoliubov inequality).

(ii) Inequality (6) is equivalent to: for all $x \in M_{n}$

$\left|\omega\left(\left[x, x^{*}\right]\right)\right|^{2} \leqq \frac{1}{2} \omega\left(x^{*} x+x x^{*}\right) \omega\left(\left[x^{*},[H, x]\right]\right)$.

(iii) Inequality (6) does not imply inequality (5).

Proof. (i) and (iii) is proved in [1] and [6].

The proof of (ii) is completely analogous as the proof of Theorem III.1 (ii).

Finally we discuss the relation of the inequalities of above with the following well known upper bound of the Duhamel two-point function: for $x \in M_{n}$.

$$
\int_{0}^{1} d \lambda \omega\left(x^{*} e^{-\lambda H} x e^{\lambda H}\right) \leqq \frac{1}{2} \omega\left(x^{*} x+x x^{*}\right) .
$$

Theorem III.3. (i) Inequality (2) implies inequality (8).

(ii) Inequality (8) does not imply inequality (7) and vice versa.

Proof. (i) is proved in [1].

We prove (ii) by an explicit computation on $M_{2}$.

Take

$$
H=\left(\begin{array}{ll}
0 & 0 \\
0 & 1
\end{array}\right), \varrho=\left(\begin{array}{cc}
\alpha & 0 \\
0 & 1-\alpha
\end{array}\right) 0 \leqq \alpha \leqq 1 .
$$

Inequality (8) is satisfied for:

$$
\alpha_{1}=\frac{(e-3 / 2)}{e-1} \leqq \alpha \leqq \frac{e}{2(e-1)}=\alpha_{2}
$$

and inequality (7) is satisfied for

$$
\frac{1}{2} \leqq \alpha \leqq \frac{3}{4}
$$

and

$$
\frac{1}{2}<\alpha_{1}<\frac{3}{4}<\alpha_{2} .
$$


The results of this section can now be summarized in the following scheme, the numbers refer to the inequalities, the arrows are implications and the bared arrows are implications which are in general not true.

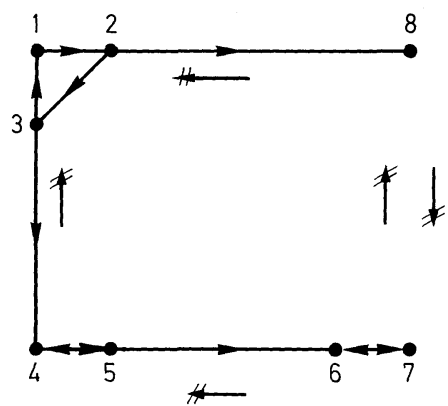

Acknowledgements. Thanks are due to B. Demoen for a very useful remark, and to Professor H. Araki for a simplication in one of the arguments.

\section{References}

1. Roepstorff,G.: Commun. math. Phys. 46, 253 (1976)

2. Fannes, M., Verbeure, A. : Commun. math. Phys. 55, 125 (1977)

3. Sewell,G.: Commun. math. Phys. 55, 53 (1977)

4. Dyson,F.J., Lieb,E.H., Simon, B.: Phase transitions in quantum spin systems with isotropic and nonisotropic interactions. J. Stat. Phys. (to appear)

5. Roepstorff, G.: Commun. math. Phys. 53, 143 (1977)

6. Naudts,J., Verbeure, A. : J. Math. Phys. 17, 419 (1976)

7 Naudts,J., Verbeure, A., Weder, R.: Commun. math. Phys. 44, 87 (1975)

Communicated by H. Araki

Received June 18, 1977; in revised form August 17, 1977 
